\title{
Sleep quality improves with endoscopic sinus surgery in patients with chronic rhinosinusitis and nasal polyposis*
}

\author{
Maria Värendh', Arne Johannisson², Harald Hrubos-Strøm³ \\ Morgan Andersson' \\ Department of Otorhinolaryngology, Skåne University Hospital and Lund University, Sweden \\ 2 Occupational Therapy and Occupational Science, Lund University, Sweden \\ 3epartment of Otorhinolaryngology, Akershus University Hospital, Lørenskog, Norway
}

Rhinology 55: 45-52, 2017

https://doi.org/10.4193/Rhino16.065

*Received for publication:

February 15, 2016

Accepted: October 2, 2016

\begin{abstract}
Background: Chronic Rhinosinusitis with Nasal Polyposis (CRSwNP) is a chronic disease that has a major impact on generic and disease-specific quality of life. Little is known about the influence of CRSwNP on sleep and what effect surgery for CRSwNP has on sleep quality. The aim of the study was to investigate sleep quality in patients with CRSwNP before and after endoscopic surgery.

Methodology: Forty-two patients filled out four validated sleep questionnaires and one sino/nasal, disease specific quality of life questionnaire before surgery and three months later. A healthy control group filled out the same questionnaires at baseline and after three months.

Results: An impact on sleep patterns was found in all sleep questionnaires and surgery clearly improved the quality of sleep. The Sino-nasal outcome test sum score decreased from median 51,5 to 26,5 . Epworth sleepiness scale showed a decline in score from score 7.5 to 6.0. Surgery also reduced the risk for obstructive sleep apnoea in 13 patients evaluated by the Berlin Questionnaire and Multivariable Apnea Prediction Index.
\end{abstract}

Conclusions: Patients with CRSwNP had impaired sleep quality, daytime sleepiness, nasal patency, and risk for sleep apnea, all of which improved after corrective surgery.

Key words: nasal mucosa, nasal surgical procedures, quality of life, respiratory tract diseases, sinusitis

\section{Introduction}

Chronic rhinosinusitis with nasal polyposis (CRSwNP) or without nasal polyposis (CRSsNP) are chronic diseases of poorly understood aetiology. These diseases are associated with other airway diseases and it has been well documented that the daily life of these patients is often severely impaired ${ }^{(1)}$. According to EPOS 2012 (European position paper on rhinosinusitis and nasal polyps 2012), a global evaluation of patients with CRSwNP should include symptom assessment, endoscopic examination, CT scan, and quality of life (QoL) evaluation. Several questionnaires have been developed for this purpose, including the Rhinosinusitis Disability Index (RDI) (2) and Sino-nasal Outcome Test (SNOT-22) $(3,4)$. However, RDI focuses mainly on daytime symptoms such as breathing through the nose. Nighttime symptoms and the consequences of a blocked nose and disturbed sleep have not been investigated thoroughly in this patient group ${ }^{(5)}$. Interestingly, even Hippocrates ${ }^{(6)}$ observed that nasal polyposis was associated with restless sleep, but few studies today have focused on nighttime problems and associated daytime symptoms in patients with nasal polyposis. Hence, there is a lack of knowledge

Abbreviations: BNSQ (Basic Nordic Sleep Questionnaire), BQ (Berlin questionnaire), CRSwNP (Chronic rhinosinusitis with nasal polyps), CRSsNP (Chronic rhinosinusitis without nasal polyps), ESS (Epworth Sleepiness Scale), FESS (functional endoscopic sinus surgery), IQR (inter quartile range), MAP (Multivariable Apnea Prediction) Index, MD (median), OR (odds ratio), OSA (obstructive sleep apnoea), SAGIC (Sleep Apnea Genetics International Consortium), SDB (sleep disordered breathing), SNOT-22 (Sino-nasal outcome test-22), QoLQ (Quality of Life Questionnaire) 
of how CRSwNP affects sleep, and any association between CRSwNP and sleep apnoea.

The gold standard for investigating patients who may suffer from sleep disordered breathing (SDB), including obstructive sleep apnoea (OSA), is polysomnography ${ }^{(7)}$. It is, however, an expensive and time consuming task to complete in patients. Sleep questionnaires are therefore often used to evaluate whether patients suffer from sleep related symptoms, are at risk of developing OSA, and/or suffer from daytime sleepiness, even if it is not as reliable as polysomnography. Thus, different sleep questionnaires have been used to select patients who are at risk of developing OSA, to detect sleep related QoL, and also to reveal sleep related symptoms.

It has been demonstrated that treatment of CRSwNP with topical steroids, oral steroids, and nasal surgery improves daytime quality of life ${ }^{(1)}$. Whether surgery also improves nighttime symptoms, patients' sleep quality, and daytime symptoms related to poor sleep, has not been thoroughly studied. The aims of this study were to determine if patients with CRSwNP suffered from reduced quality of sleep compared to a healthy population in terms of snoring, and indirect symptoms of sleep apnoea. A further aim was to investigate whether functional endoscopic sinus surgery (FESS) improved any of these symptoms in an unselected group of patients with CRSwNP.

\section{Materials and methods}

Study outline

This was an open prospective study with patients who were selected for endoscopic surgery due to nasal polyposis from September of 2013 to April of 2014 at the Department of Otorhinolaryngology, Skåne University Hospital, Sweden.

\section{Subjects}

45 patients ( 12 females and 33 males) participated. All patients suffered from severe nasal polyposis with grade 2-3 polyposis according to the Lildholdt scale and were offered surgery ${ }^{(8,9)}$. Lildholdt grade 2 (moderate polyposis) is recognized by "medium sized polyps reaching between the upper and lower edges of the inferior turbinate". Grade 3 (severe polyposis) is recognized by "large polyps reaching below the lower edge of the inferior turbinate". Preoperatively, patients were asked to answer four different validated sleep questionnaires and one sino/nasal QoL questionnaire. All patients underwent preoperative endoscopic nasal examination for polyp grading and spirometry, as well as a CT-scan. Approximately three months after sugery, patients were re-examined with the same questionnaires and a second spirometry was performed. All patients gave oral and written informed consent. Patients' files were retrieved to secure medication, smoking status, and co-morbidities (hypertension, asthma and allergies).

Inclusion criteria included: being over 18 years of age; surgery planned for nasal polyposis; being able to understand instructions and questions in Swedish; and able to fill out forms without major help. Patients were not included if pregnant.

The recruitment was consecutive and only a few patients were missed for inclusion due to clinicians forgetting to include them. An additional three patients were planned to participate in the study, but one dropped out due to an unwillingness to undergo surgery, and two were delayed for surgery and could therefore not be included during the study period. Median time between baseline and follow up was 23 weeks (9-44 weeks).

To assess reliability, 37 healthy subjects were prospectively asked to fill out the questionnaires three months apart during the same period as the study was completed.

\section{Questionnaires}

The sino-nasal outcome test SNOT-22 item version is a validated disease specific quality of life questionnaire focusing on symptoms related to CRS+/-NP. The SNOT-20 was developed by Piccirillo et al. ${ }^{(3)}$. In 2009, Hopkins et al. added two questions about taste and smell ${ }^{(4)}$. The Swedish version of the SNOT-22 was first validated in $2011^{(10)}$. Healthy subjects report in SNOT-22 a total score between 0 and $8^{(11,12)}$.

\section{The Sleep Apnea Genetics International Consortium (SAGIC)} (http://www.med.upenn.edu/sleepctr/SAGIC.shtml) has developed a set of sleep questionnaires including the Epworth Sleepiness Scale (ESS) ${ }^{(13)}$, the Basic Nordic Sleep Questionnaire (BNSQ) ${ }^{(14)}$, the Berlin Questionnaire (BQ) ${ }^{(15)}$, and the Multivariable Apnea Prediction (MAP) index ${ }^{(16)}$. The SAGIC group has made minor changes in the sleep questionnaires, as described below. This set of questionnaires was translated by a professional interpreter into Swedish, double checked by a medical doctor and re-translated into English by another professional interpreter according to standardized guidelines ${ }^{(17)}$. Again a medical doctor revised it. Questions about former surgery were included from a previous study by the authors ${ }^{(18)}$.

The ESS, that aims to measure daytime sleepiness, was developed by Murray Johns in $1991^{(13)}$. Subjects are asked to rate their probability of falling asleep on a scale from 0 to 3 for eight different situations that are encountered during daily living (maximum score 24). A number from 0 to 10 is considered normal, scores from 11-15 are indicative of mild to moderate daytime sleepiness, whereas scores higher than 15 indicate severe daytime sleepiness. Healthy controls who do not snore tend to have an average score of 6-7 ${ }^{(13,19,20)}$.

The BNSQ surveys perception of usual sleep quality, sleep latency, number of awakenings per night, naps per day and symptoms of poor quality of sleep in the past three months ${ }^{(14)}$. The SAGIC BNSQ version also contains an additional question about being tired in front of the computer; and an additional 
"Don't know" alternative has been added to all categorical items. Other categories are "Never", "Rarely: less than once a week", "Frequently: 3-4 times a week and "Always: 5-7 times a week". We also calculated BNSQ-symptoms as a sum.

The $B Q^{(15)}$ is a screening tool used for the identification of patients who may be at high risk of OSA. The index gives either 0 for no risk of OSA or 1 for risk of OSA. Healthy non-snorers have 0 risk. The questionnaire consists of 11 questions separated into 3 categories: episodes of snoring and cessation of breathing while sleeping; daytime sleepiness; and incidence of high blood pressure. When two or more categories are classified as positive, the patient is considered to be at high risk for OSA. The SAGIC version of the BQ has also added a "don't know" category to all items with categories of "yes" and "no". Moreover, the question "Do you have high blood pressure?" has been changed to "Did you ever get a diagnosis of hypertension from a doctor?" If yes, the categories are: "Are you on anti-hypertensive medication at the moment? No/Yes".

The MAP index ${ }^{(16)}$ predicts OSA risk using demographic data and self-reported apnoea symptoms. Three frequency questions as well as gender, age, height and weight are used to produce a MAP index between 0 (low risk) and 1 (high risk). The three questions determine the frequency at which the patient experienced loud snoring, snoring or gasping, cessation of breathing, or struggling for breath in the last month. Together, the questions produce a score referred to as index 1 . A cut-off point of indication for a sleep registration has been suggested by Maislin to be $0.5^{(16)}$. This value has been shown to have a sensitivity of 0.88 and a specificity of 0.55 and a positive predictive value of 0.75 . Bjornsdottir ${ }^{(21)}$ has chosen 0.75 for cut off in a clinical population since 0.5 is more appropriate for use in a general population. Accordingly, we chose 0.75 to separate the patients into low risk and high risk of OSA, since our patients have disease in their upper airways and are at risk of developing OSA. Twentytwo patients answered all three MAP questions before and after surgery and these patients were used for the results within. No imputations were used in MAP.

\section{Measurements and equipment}

An electronic spirometer was used for spirometry assessment in a standing position (Micro lab 3300, Micromedial Ltd, Rochester, England). Patients were told to take a deep breath and blow out as hard as they could. Then one inhalation of a bronchodilator, Oxis $^{\circledast}$ (Formoterol $^{\circledR} 4.5 \mu \mathrm{g}$, AstraZeneca AB) was given and 15 minutes later a second assessment was made in the same way. Percentage of the expected value of forced expired volume in one second was used for analysis (FEV1). Patients who reported a doctor diagnosis of asthma/COPD and were on asthma/COPD medication were classified as suffering from asthma/COPD.

\section{Polyp size assessments}

CT-scans were completed in all patients prior to surgery and the size of the polyps graded according to the Lund-Mackay scoring

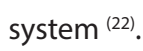

\section{Surgery}

Patients underwent endoscopic sinus surgery under general anesthesia according to our clinical routine. All patients were operated with microdebrider in day-care settings using intravenous anesthesia. Five different well-established ENT surgeons completed the surgeries; but the majority were done by one surgeon (MA). The surgeons were well informed about the study and gave written consent. Postoperatively, nasal packing was used for one to three days and nasal washings with saline for a restricted time was recommended. Oral corticosteroid treatment was given for a limited time up to three weeks according to the EPOS guidelines (1). All patients were pre- and postoperatively treated with local corticosteroids as a routine treatment for nasal polyposis. No extra oral corticosteroids except for the initial postoperative treatment were given, and no other pharmacotherapy was changed between the two assessments.

Approval by the regional ethical committee at Lund University was granted for this study (Dnr 2013/491).

\section{Data analysis and statistical procedures}

Data on nominal levels were presented as frequencies and percentages. No decimals were used for the percentages. The Chi-squared test was used in comparisons between nominal data. In case of insufficient number of expected count; Fisher's exact test was used. Ordinal data were presented as the median and interquartile range (IQR). All values are given as median unless otherwise stated. Differences between groups were calculated by means of Mann-Whitney U test. Wilcoxon's Signed Rank test was used for comparisons over time. Spearman's rank correlation $\left(r_{s}\right)$ was used when measuring associations. The BQ and the MAP Index were computed according to the manufacturer's instructions. For the other instruments, the variables were presented one by one or/and summarized to scores. The actual number of observations is given as ratios, as the number of patients who have given a certain answer/total number, and as the percent of the number who provided an answer to that specific question.

The binary logistic regression was completed with the enter method, i.e. all predictors were included in the calculations. Imputations: Missing data in any ordinal variable were replaced with the median data value given by each subgroup; the polyposis and the controls. The imputations were used when presenting and comparing group data. When presenting data regarding change of risk over time in $\mathrm{BQ}$ and MAP, non-imputated individual data were used. 
Table 1. Characteristics of the study population separated into Asthma/ COPD (Chronic obstructive pulmonary disease) and Non-asthma/COPD groups, as well as healthy controls. Values are given as median, min-max and percentage of the whole study group. Lund-Mackay CT-staging evaluation smoking prevalence, OSA (Obstructive Sleep Apnoea), and hypertension are presented in numbers and proportions of patients. In the female group, asthma was more common (9/12: 75\%) than in the male group (15/30: 50\%). Other abbreviations used: BMI (Body Mass Index) and CT (computed tomography).

\begin{tabular}{|c|c|c|c|c|}
\hline Subjects & $\begin{array}{l}\text { Asthma/ } \\
\text { COPD }\end{array}$ & $\begin{array}{l}\text { Non-Asth- } \\
\text { ma/COPD }\end{array}$ & Controls & All patients \\
\hline & $\begin{array}{l}N=24 \\
(57 \%)\end{array}$ & $\begin{array}{l}N=18 \\
(43 \%)\end{array}$ & $\mathrm{N}=38$ & $\mathrm{~N}=42$ \\
\hline $\begin{array}{l}\text { Female } \\
\text { (Male) }\end{array}$ & $9(15)$ & $3(15)$ & $22(16)$ & $12(30)$ \\
\hline Age (years) & $51(31-76)$ & $50(28-71)$ & $40(28-65)$ & $50(28-76)$ \\
\hline BMI & $26.0(19-35)$ & $27.0(19-31)$ & - & $26.2(19-35)$ \\
\hline Smoker & 2 & 4 & 8 & $6(14 \%)$ \\
\hline OSA & 1 & 1 & 1 & $2(5 \%)$ \\
\hline $\begin{array}{l}\text { Hyperten- } \\
\text { sion }\end{array}$ & 8 & 2 & 2 & $10(24 \%)$ \\
\hline $\begin{array}{l}\text { Lund- } \\
\text { Mackay }\end{array}$ & $17.5(6-24)$ & $13.5(8-24)$ & - & $15.5(6-24)$ \\
\hline
\end{tabular}

In all analyses, SPSS 22.0 was used. A two-sided p-value $<0.05$ was considered significant in all calculations. P-values (significant or not) are presented for all comparisons.

\section{Results}

Baseline data

Forty-two patients participated (12 females and 30 males). Their ages ranged between 28 to 76 years (median: 50 years). Ten patients suffered from pollen allergy and all ten also reported other allergies (house dust mite, animal dander). Another 4 patients reported allergies other than pollen and in total, 14 patients reported an allergy of any kind. There were 34 patients living in a household with at least one other person. Patients' characteristics are described in Table 1, showing that patients who had a diagnosis of asthma/COPD were quite similar to the non-asthma/COPD in terms of age and BMI. In both groups, one patient had a diagnosis of obstructive sleep apnoea. Asthma was more common in females (9/12: 75\%) than in males (15/30: $50 \%)$. Patients with asthma and/or COPD had a tendency towards a more severe polyposis compared to the non-asthma/ COPD patients according to the CT staging (17.5 vs. 13.5 , $\mathrm{p}=0.058$ ).

\section{SNOT-22 questionnaire}

Questionnaire derived pre- and postoperative sum scores are
Table 2. Pre- and post operative median and interquartile range for SNOT- 22 (Sino Nasal Outcome Test-22), BNSQ (Basic Nordic Sleep Questionnaire), and ESS (Epworth Sleepiness Scale). There was a significant improvement in SNOT, ESS, and BNSQ three months after surgery. Significant values in bold.

\begin{tabular}{lllll|}
$\begin{array}{l}\text { Question- } \\
\text { naire }\end{array}$ & Pre Op & Post op & Difference & p-value \\
\hline & Med (IQR) & Med (IQR) & Med (IOR) & \\
\hline SNOT-22 & $51.5(37)$ & $26.5(15)$ & $\mathbf{- 1 8 . 0 ( 2 7 )}$ & $<\mathbf{0 . 0 0 1}$ \\
\hline ESS & $7.5(6)$ & $6.0(5)$ & $\mathbf{- 1 . 0}(4)$ & $\mathbf{0 . 0 4 8}$ \\
\hline BNSQ & $11.0(8)$ & $8.0(8)$ & $\mathbf{- 2 . 0}(4)$ & $\mathbf{0 . 0 0 1}$ \\
\hline
\end{tabular}

presented in Table 2. The SNOT-22 sum score declined from 51,5 to 26,5 . Single item analyses showed that 38 out of a total of 42 patients (91\%) suffered from major problems with blocked nose, rated 4 (severe) or 5 (as bad as it can be) prior to surgery, while just five patients reported major problems with blocked nose (12\%) after surgery. Before surgery, 23/40 (55\%) patients reported major problems with "nose blowing" (score 4-5). After surgery, 5/30 (12\%) patients reported major problems with nose blowing (score 4-5).

Patients who reported major problems with sneezing (score 4-5) had a 12.90 higher OR of reporting "Lack of a good night's sleep" when compared to patients without sneezing (C.I. 95\% 2.02-70.03, $\mathrm{p}=0.006)$. Sneezing was reported as a major problem in $14 / 29$ (48\%) patients before surgery and just 5/29 (17\%) after surgery.

The question: "Lack of good night's sleep" was scored as a major problem in 10/31 (32\%) compared to $5 / 31(16 \%)$ after surgery $(p<0.001)$. When looking at patients aged over 60 compared to less than 60 years, the younger patients scored higher (11/30, $63 \%)$ compared to older patients $(2 / 11,18 \%)$ with respect to "Lack of good night's sleep".

A large proportion of the patients (17/38, 45\%) reported severe nasal symptoms prior to surgery ("Need to blow nose", "Nasal blockage", "Sneezing", and "Runny nose" (score 4-5 in all four)). There was no significant correlation between CT-stages and the total SNOT-22 score $r s=0.033, p=0.863$.

\section{Sleep questionnaires}

In Table 2, the total scores for ESS and BNSQ are depicted as well as SNOT-22, as mentioned earlier. Total ESS scores decreased from 7.5 pts. to 6.0 pts. $(p<0.05)$ after surgery. Three patients scored over 15 before surgery, a score considered as excessive daytime sleepiness. Twelve patients scored over 10. Eleven patients (24\%) scored 7 or 8 . Regarding symptoms of poor sleep quality; BNSQ symptoms were calculated as a total sum and demonstrated a similar and clear decrease from 11.0 pts. to 8.0 pts. 


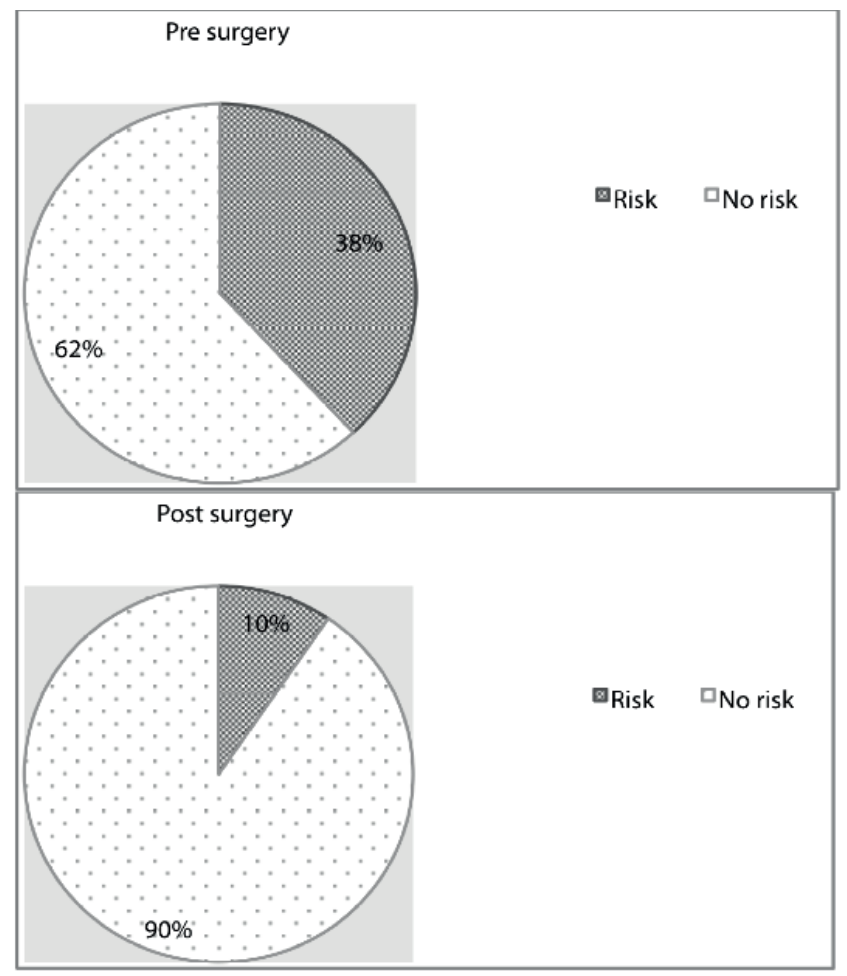

Figure 1. Results of the Berlin Questionnaire depicted in percentage of the whole patient group $(n=42)$. The risk changed in 13 patients from being at risk of obstructive sleep apnoea (OSA) to non-risk after surgery. One patient went from non-risk to OSA risk. Three patients who were at risk of developing OSA before surgery were still at risk after surgery.

$(p<0.01)$ after surgery. Specific BNSQ items have been presented in Table 3. There was a significant decrease in symptoms of poor sleep quality in the item "I wake up with a dry mouth" from 1.52 to $1.0(p<0.001)$.

Other Sleep Questionnaires: Berlin Questionnaire and MAP Using the $B Q$, subjects could be identified who are at risk of developing obstructive sleep apnoea (OSA). According to the $B Q$, surgery changed 13 of the patients from being at risk of OSA to a non-risk group after surgery, but one patient went from nonrisk to OSA risk after surgery. Three patients who were at risk of developing OSA before surgery were still at risk after surgery (Figure 1). Of the 13 patients who moved from risk to non-risk after surgery, nine were snorers at baseline. All 13 patients were "tired, fatigued, or not up to par" 3-4 times per week or nearly every day before surgery. The question "Do you snore?" was answered "yes" by 26 (62\%) before surgery and 15 (36\%) after surgery $(p=0.072)$.

MAP Index predicts OSA risk using demographic data and selfreported apnoea symptoms. The results are shown in Table 2. When using 0.75 as the cut off for being at risk, 13 patients went from risk to no risk after surgery. Four patients went from no-risk
Table 3. BNSQ (Basic Nordic Sleep Questionnaire), symptoms before (pre operative) and 3 months after (post operative) surgery (median and interquartile range). As seen, the individual responses improve after surgery. Significant values in bold.

\begin{tabular}{|c|c|c|c|}
\hline BNSQ & Pre op & Post op & p-value \\
\hline & $\begin{array}{l}\text { Med } \\
\text { (IQR) }\end{array}$ & $\begin{array}{l}\text { Med } \\
\text { (IQR) }\end{array}$ & \\
\hline 1) I wake up with a headache & $1.0(1.0)$ & $1.0(1.0)$ & 0.224 \\
\hline 2) I wake up with a dry mouth & $3.0(2.0)$ & $1.0(2.0)$ & $<0.0001$ \\
\hline $\begin{array}{l}\text { 3) I wake up because of hot } \\
\text { flashes }\end{array}$ & $0.0(1.0)$ & $0.0(1.0)$ & 0.025 \\
\hline $\begin{array}{l}\text { 4) I sweat/perspire excessively } \\
\text { during the night }\end{array}$ & $1.0(2.0)$ & $1.0(1.0)$ & 0.360 \\
\hline $\begin{array}{l}\text { 5) I toss, turn and thrash exces- } \\
\text { sively during the night }\end{array}$ & $2.0(3.0)$ & $2.0(1.0)$ & 0.374 \\
\hline $\begin{array}{l}\text { 6) I sweat/perspire excessively } \\
\text { during the day }\end{array}$ & $1.0(4.0)$ & $1.0(1.0)$ & 0.299 \\
\hline $\begin{array}{l}\text { 7) I get heartburn after lying } \\
\text { down }\end{array}$ & $0.0(1.0)$ & $0.0(1.0)$ & 1.00 \\
\hline 8) I get heartburn during the day & $0.0(1.0)$ & $1.0(1.0)$ & 0.273 \\
\hline $\begin{array}{l}\text { 9) I fall asleep involuntarily during } \\
\text { the day e.g. when I take a break } \\
\text { from my work }\end{array}$ & $0.0(0.0)$ & $0.0(0.0)$ & 0.942 \\
\hline $\begin{array}{l}\text { 10) I feel very sleepy during the } \\
\text { day. e.g. when I sit in front of the } \\
\text { computer }\end{array}$ & $1.0(2.0)$ & $1.0(2.0)$ & 0.073 \\
\hline
\end{tabular}

to risk after surgery.

\section{Specific question}

The question: "Do you suffer from a blocked nose at night?" was answered yes by $21 / 27$ (75\%) before surgery and by $8 / 24$ (33\%) after surgery.

To the general question: "How satisfied/unsatisfied are you with your current sleep? ( $0=$ very satisfied, to $4=$ very dissatisfied) $13 / 42$ (36\%) patients reported unsatisfied (score 3-4) with sleep before surgery, which improved to $3 / 34$ (9\%) after surgery. The number of patients who were satisfied with their sleep (score: 0 - 2) increased from $17 / 42(40 \%)$ to $27 / 34(79 \%)$ after surgery $(\mathrm{p}<0.001)$.

\section{Comparison between the questionnaires}

A relationship was noted between the answers in SNOT-22 and BNSQ ( $r s=0.659, p<0.001$ ), as well as between SNOT-22 and ESS ( $r s=0.362, p=0.019$ ).

\section{Asthma}

Twenty-nine patients were investigated by spirometry before and after surgery, but the rest could not be motivated or find the time to be investigated before and after surgery. However, 
Table 4. Healthy control individuals answering the same questionnaires 3 months apart. Data presented in median and interquartile range. As seen there were no differences in the responses at baseline compared to three months later. SNOT-22 (Sino Nasal Outcome Test-22), ESS (Epworth Sleepiness Scale), and BNSQ (Basic Nordic Sleep Questionnaire) illustrated.

\begin{tabular}{lllll}
$\begin{array}{l}\text { Question- } \\
\text { naire }\end{array}$ & Baseline & $\begin{array}{c}\text { After 3 } \\
\text { months }\end{array}$ & Difference & p-value \\
\hline SNOT-22 & $5.5(13.0)$ & $8.0(12.0)$ & $0.5(10.0)$ & 0.08 \\
\hline ESS & $5.0(5.0)$ & $5.0(5.0)$ & $0.0(2.0)$ & 0.786 \\
\hline BNSQ & $3.0(7.0)$ & $3.0(6.0)$ & $0.0(3.0)$ & 0.775 \\
\hline
\end{tabular}

surgery did not change FEV1\%: 94 (IQR 26) vs. 90 (IQR 24.2) after surgery, $p=322$. This was true both for the asthmatic/COPD group at 86.7 (IQR 32) before and 75.4 (IQR 23) after surgery $(p=0.455)$ and for the non-asthma/COPD group at 101 (IQR 21.3) before vs. 92 (IQR 20.8) after surgery ( $p=0.433)$.

There was no difference in SNOT-22 $(p=0.94)$, ESS $(p=1)$, or BNSQ $(p=0.654)$ between asthmatics and non-asthmatics before or after surgery.

\section{Healthy controls}

Table 4 shows the questionnaire sum scores from 37 healthy volunteers. No significant differences in any of the answers could be found between the two occasions in SNOT-22 ( $p=0.08)$, ESS $(p=0.786), B N S Q(p=0.775)$, and Berlin (no risk before $(100 \%)$ and with one person scoring a risk after 3 months).

\section{Discussion}

In this prospective open study of an unselected group of patients suffering from CRSwNP, we found that a majority had reduced sleep quality compared to a healthy population, with associated night- and daytime symptoms. Endoscopic surgery improved these symptoms.

Our objective was to look into how patients with CRSwNP evaluated their quality of sleep and the consequences of poor quality sleep. We also assessed snoring and indirect symptoms of sleep apnoea, and whether functional endoscopic sinus surgery (FESS) improved any of these symptoms in an unselected group of patients undergoing surgery. Several questionnaires were used to evaluate sleep quality and associated daytime symptoms. The validated rhinological QoL questionnaire, SNOT-22, which also includes some sleep questions, found a clear relationship with some of the sleep questionnaires. In general, sleep questionnaires are useful tools for sleep categorization even though polysomnography is the golden standard for measurement of sleep apnoea. Questionnaires do of course not give an objective evaluation of patients' sleep, in contrast to polysomnography. However, a sleep registration was not possible to complete in the present study. All sleep questionnaires pointed in the same direction, namely that patients reported poor sleep before surgery and that surgery improved their sleep. By using the questionnaires on a healthy population during the same time of year, we demonstrated good test-retest reliability. A test-retest is one possible way to evaluate if similar results are reproduced under the same methodological conditions, but at different times ${ }^{(23)}$. SNOT-22 showed an improvement from being very abnormal to less abnormal. Before surgery, the patients suffered from major problems with nasal symptoms and reduced quality of life ${ }^{(11,12)}$. Patients in the present study reported a score of 26.5 after surgery, in contrast to healthy subjects who score $0-8{ }^{(10,11)}$, a finding demonstrating that even though surgery improved quality of life, CRSwNP still has a major impact on daily life. However, the improvement in nasal scoring after surgery most likely remains of major importance to the patients.

With regard to the sleep questionnaires, we found a daytime sleepiness prior to surgery, as evaluated by ESS, which was similar to a normal value for a healthy person (6.6) ${ }^{(19)}$. Daytime sleepiness decreased with surgery from median score 7.5 to 6.0. Surgery has an impact on their level of tiredness. These patients are tired during the day at the same level reported by healthy people, but they are less tired after surgery than they were before. Healthy people score between 0 and 7 in ESS ${ }^{(13,21)}$. Any improvement, within or from above normal score, is in any case of great value for the individual. Jiang et al. ${ }^{(24)}$ found a high percentage of patients with CRSsNP having daytime sleepiness and they found a correlation between sleepiness and nasal blockage.

Since BNSQ-symptoms were calculated as a sum for the first time, there are no references for comparison, but there is an improvement compared to before surgery. When calculating the symptoms in BNSQ as a sum we get a score that gives a value for the consequences of poor sleep in the patients. The consequences of poor sleep are of great concern to patients and an improvement is most likely of great value for the individual patient.

The BQ gives a risk estimate of obstructive sleep apnoea. Interestingly, the $B Q$ showed that a large proportion of the patients (38\%) were actually at risk of developing sleep apnoea, but surgery reduced the risk for 13 patients. The MAP Index also provides an estimate of risk based on snoring and apnoea questions together with gender, age, height, and weight. The improvement in the MAP estimate was similar to the improvement in BQ risk. A large proportion of the patients demonstrated a risk for OSA prior to surgery, which was reduced by surgery. We found that the patients who improved were all tired or "not up to par" to a major extent during a normal week before surgery. 
A correlation between nasal symptoms and sleep was observed. Patients who suffered from sneezing indicated a higher risk of waking up feeling tired.

Healthy people breathe through the nose to a major extent while sleeping ${ }^{(25)}$. It has been suggested that the negative pressure reflex is then stimulated by nasopharyngeal breathing, and through afferent nerves, stimulates the upper airway dilator muscle activity ${ }^{(26,27)}$. In the presence of nasal polyps, or other reasons for nasal congestion, the patients make a shift to oral breathing and the negative pressure reflex is not stimulated, which can result in an increased risk of collapsed airways leading to apnoea ${ }^{(28-30)}$. The nose seems to be an important factor for a good night's sleep. High nasal resistance could be considered as an important contributing factor in the pathogenesis of OSA in general. Any factor that produces nasal obstruction could lead to an increase in negative pressure in the upper airway. Factors that cause nasal obstruction, like nasal polyposis or hypertrophy of the inferior turbinate, have been associated with snoring and daytime sleepiness ${ }^{(31)}$. Some clinical studies have reported previously subjective and objective improvement of OSA after nasal surgery like rhinoplasty, septoplasty, turbinectomy, and polypectomy ${ }^{(32)}$. The patients in our study were clinically normal patients suffering from nasal polyposis. Hence, awareness of the risk for nasal polyposis patients to have or being at risk for obstructive sleep apnoea is highly warranted. Moreover, maybe surgery should be considered more often to reduce this risk.

Our finding of a high proportion of co-morbid asthma (57\%) is in line with a previous study from Promsopa and colleagues ${ }^{(33)}$ and we wanted to see whether FESS also improved lung function. If the asthma improved, it could have been a confounding factor also for the improvement of sleep related symptoms. The observation that the asthmatics in our study most likely had a more severe polyposis disease as assessed by CT scan, than the non-asthmatics, indicates support for a connection between asthma and nasal polyposis, as shown in earlier studies ${ }^{(34)}$. The observation that CT-stages did not correlate with patients' answers in SNOT-22 is in line with other types of studies looking at the relationship between objective findings comparing subjective data ${ }^{(35)}$. In contrast to other studies ${ }^{(1)}$, although with some conflicting results ${ }^{(36)}$, our patients with asthma did not improve their lung function as measured by spirometry three months after surgery. The number of patients who were investigated with spirometry was unfortunately low and the dropout may have caused a bias. It could be speculated that specific asthma questionnaires could have revealed improvements in this group or that any specific measurement of bronchial hyperactivity with bronchial provocations or measurements of exhaled nitric oxide would have been a better method to observe improvements in lung function after surgery. The objective of this study, however, was not to address the question of asthma improvements after endoscopic surgery.

Concerning allergies, the number of patients with any kind of allergy was low and the surgery was completed during the winter months rather than during the pollen season.

\section{Strengths and limitations of the study}

Although the number of patients in this study was fairly small, a clear reduction of sleep-associated symptoms was seen following surgery. It can be speculated that if a larger number of patients had been included, a larger impact on all symptoms might have been observed, but during the given timeline, it was not possible to recruit more patients. A selection bias is a problem when it comes to clinical studies, but all patients who were planned for surgery (except three excluded) also completed the whole procedure. Hence, the group of patients may well represent a fairly normal group of nasal polyposis patients. The study has an open design, which may hamper the results. A double blind placebo controlled randomised study would have been ideal, but for obvious reasons it is not possible to perform sham surgery. Questionnaires may have limits in how much they correlate with sleep registrations ${ }^{(20)}$ and it is a limit of this study that a polysomnography was not completed. However, a sleep registration was not possible in the present study. The number of patients who were investigated with spirometry was unfortunately low and the dropout could have given a bias to the results.

In future studies it would be of great value if the shortfalls of this study concerning objective measurements could be improved to allow the possibility of discriminating between nasal patency before and after surgery.

\section{Conclusion}

In conclusion the study has demonstrated that patients with chronic rhinosinusitis with nasal polyposis have impaired sleep and that endoscopic sinus surgery can improve these sleep impairments. We propose that from a clinical point, it is important to address the sleep related symptoms of patients and be aware that those with polyposis are at risk of developing obstructive sleep apnoea syndrome. In general, surgery had a clear impact on nose symptoms, sleep symptoms, daytime drowsiness, and snoring scores.

\section{Acknowledgements}

We thank Lena Glantz Larsson and Charlotte Cervin Hoberg, Anna Karin Bastos, and Ulf Höckerfelt for helpful assistance with spirometry and data collection. We are also grateful to Ulla Johannisson who helped with data and Patti and George Holmgren who helped with the manuscript. A special thank you is addressed to Robin L. Anderson for invaluable support in the final writing process. 


\section{Authorship contribution}

MV: Planned the study, organized the collection of data, performed statistical calculations and analysis, main writer of manuscript. AJ: Performed statistical calculations and analysis. HHS: Analysed results and wrote the manuscript. MA: planned and organized the study. Also took a major part in analysing results and writing the manuscript.

\section{Conflict of interest}

Authors declare no conflict of interest.

\section{References}

1. Fokkens WJ, Lund VJ, Mullol J, et al. EPOS 2012: European position paper on rhinosinusitis and nasal polyps 2012. A summary for otorhinolaryngologists. Rhinology. 2012;50(1):1-12.

2. Benninger MS, Senior BA. The development of the Rhinosinusitis Disability Index. Arch Otolaryngol Head Neck Surg. 1997;123(11):1175-9.

3. Piccirillo JF, Merritt MG, Richards ML. Psychometric and clinimetric validity of the 20-Item Sino-Nasal Outcome Test (SNOT-20). Otolaryngol Head Neck Surg. 2002;126(1):41-7.

4. Hopkins C, Gillett S, Slack R, Lund VJ, Browne JP. Psychometric validity of the 22-item Sinonasal Outcome Test. Clin Otolaryngol. 2009:34(5):447-54.

5. Alt JA, Smith TL. Chronic rhinosinusitis and sleep: a contemporary review. Int Forum Allergy Rhinol. 2013;3(11):941-9.

6. Freind J, Frewin R. Johannis Freind Commentarii novem de febribus ad Hippocratis de Morbis popularibus libros primum et tertium accomodati. 1717;3-236.

7. Collop NA, Anderson WM, Boehlecke B, et al. Clinical guidelines for the use of unattended portable monitors in the diagnosis of obstructive sleep apnea in adult patients. Portable Monitoring Task Force of the American Academy of Sleep Medicine. J Clin Sleep Med. 2007;3(7):737-47.

8. Lildholdt T, Rundcrantz $H$, Bende $M$, Larsen K. Glucocorticoid treatment for nasal polyps. The use of topical budesonide powder, intramuscular betamethasone, and surgical treatment. Arch Otolaryngol Head Neck Surg. 1997;123(6):595-600.

9. Johansson L, Åkerlund A, Holmberg K Melen I, Stierna P, Bende M. Evaluation of Methods for Endoscopic Staging of Nasal Polyposis. Acta Otolaryngol. 2000;120(1):72-

10. Sahlstrand-Johnson P, Ohlsson B, Von Buchwald C, Jannert M, Ahlner-Elmqvist M. A multi-centre study on quality of life and absenteeism in patients with CRS referred for endoscopic surgery. Rhinology. 2011;49(4):420-8

11. Yeolekar. A Study of SNOT 22 Scores in Adults with no Sinonasal Disease. Rhinolaryngo-otologies. 2013;1:6-10.

12. Gregório LL, Andrade JS, Caparroz FA, Saraceni Neto P, Kosugi EM. Influence of age and gender in the normal values of Sino Nasal Outcome Test-22. Clin Otolaryngol. 2015;40(2):115-20.

13. Johns MW. A new method for measuring daytime sleepiness: the Epworth sleepiness scale. Sleep. 1991;14(6):540-5.
14. Partinen, Gislason. Basic Nordic Sleep Questionnaire (BNSQ): a quantitated measure of subjective sleep complaints. J Sleep Res. 1995;4(S1):150-5.

15. Netzer NC, Stoohs RA, Netzer CM, Clark K Strohl KP. Using the Berlin Questionnaire to identify patients at risk for the sleep apnea syndrome. Ann Intern Med. 1999;131(7):485-91.

16. Maislin G, Ahmed MM, Gooneratne N, et al Single slice vs. volumetric MR assessment of visceral adipose tissue: reliability and validity among the overweight and obese. Obesity (Silver Spring). 2012;20(10):2124-32.

17. Fayers PM, Hopwood P, Harvey A, Girling DJ, Machin D, Stephens R. Quality of life assessment in clinical trials--guidelines and a checklist for protocol writers: the U.K. Medical Research Council experience. MRC Cancer Trials Office. Eur J Cancer. 1997;33(1):20-8.

18. Värendh $M$, Berg S, Andersson M. Longterm follow-up of patients operated with Uvulopalatopharyngoplasty from 1985 to 1991. Respir Med. 2012;106(12):1788-93.

19. Bjornsdottir E, Keenan BT, Eysteinsdottir B et al. Quality of life among untreated sleep apnea patients compared with the general population and changes after treatment with positive airway pressure. J Sleep Res. 2015;24(3):328-38.

20. Hrubos-Strøm H, Randby A, Namtvedt SK, et al. A Norwegian population-based study on the risk and prevalence of obstructive sleep apnea. The Akershus Sleep Apnea Project (ASAP). J Sleep Res. 2011;20(1 Pt 2):162-70.

21. Björnsdóttir $E$, Janson C, Gíslason $T$, et al. Insomnia in untreated sleep apnea patients compared to controls. J Sleep Res. 2012;21(2):131-8

22. Lund VJ, Mackay IS. Staging in rhinosinusitus. Rhinology. 1993;31(4):183-4.

23. Rousson V, Gasser T, Seifert B. Assessing intrarater, interrater and test-retest reliability of continuous measurements. Stat Med. 2002;21(22):3431-46.

24. Jiang RS, Liang $\mathrm{KL}$, Hsin $\mathrm{CH}$, Su MC. The impact of chronic rhinosinusitis on sleep-disordered breathing. Rhinology. 2016;54(1):75-9.

25. Wilhelm CP, deShazo RD, Tamanna S, Ullah MI, Skipworth LB. The nose, upper airway, and obstructive sleep apnea. Ann Allergy Asthma Immunol. 2015;115(2):96-102.

26. Horner R, Innes J, Holden H. Afferent pathway (s) for pharyngeal dilator reflex to negative pressure in man: a study using upper airway anaesthesia. J Physiol 1991:436(1):31-44.

27. Pierce R, White D, Malhotra A. Upper airway collapsibility, dilator muscle activation and resistance in sleep apnoea. Eur Respir J. 2007;30(2):345-53.

28. Fitzpatrick MF, McLean H, Urton AM, Tan A, O'Donnell D, Driver HS. Effect of nasal or oral breathing route on upper airway resistance during sleep. Eur Respir J. 2003;22(5):827-32.

29. Fitzpatrick M, Driver $H$, Chatha N, Voduc N, Girard A. Partitioning of inhaled ventilation between the nasal and oral routes during sleep in normal subjects. J Appl Physiol; 2003;94(3):883-90.

30. Meurice J, Marc I, Carrier G. Effects of mouth opening on upper airway collapsibility in normal sleeping subjects. Am J Respir Crit Care Med. 1996;153(1):255-9.

31. Li H-YY, Lin Y, Chen N-HH, Lee L-AA, Fang T-JJ, Wang P-CC. Improvement in quality of life after nasal surgery alone for patients with obstructive sleep apnea and nasal obstruction. Arch Otolaryngol Head Neck Surg. 2008;134(4):429-33.

32. Dayal VS, Phillipson EA. Nasal surgery in the management of sleep apnea. Ann Otol Rhinol Laryngol. 1985;94:550-4.

33. Promsopa C, Kansara S, Citardi MJ, Fakhri S, Porter P, Luong A. Prevalence of confirmed asthma varies in chronic rhinosinusitis subtypes. Int Forum Allergy Rhinol; 2015;6(4):373-7.

34. Pearlman AN, Chandra RK, Chang D, et al. Relationships between severity of chronic rhinosinusitis and nasal polyposis, asthma, and atopy. Am J Rhinol Allergy. 2009;23(2):145-8.

35. Park D-Y, Lee E, Kim J, Kim Y, Jung C, Kim K-S Correlation between symptoms and objective findings may improve the symptombased diagnosis of chronic rhinosinusitis for primary care and epidemiological studies. BMJ Open. 2015;5(12):e009541.

36. Rix I, Håkansson K, Larsen CG, Frendø M von Buchwald C. Management of chronic rhinosinusitis with nasal polyps and coexisting asthma: A systematic review. Am J Rhinol Allergy. 2015;29(3):193-201.

\section{Maria Värendh, MD}

Department of Otorhinolaryngology

Skåne University Hospital

SE- 22185 Lund

Sweden

Phone: $+46-46171000$

E-mail: maria.varendh@med.lu.se 